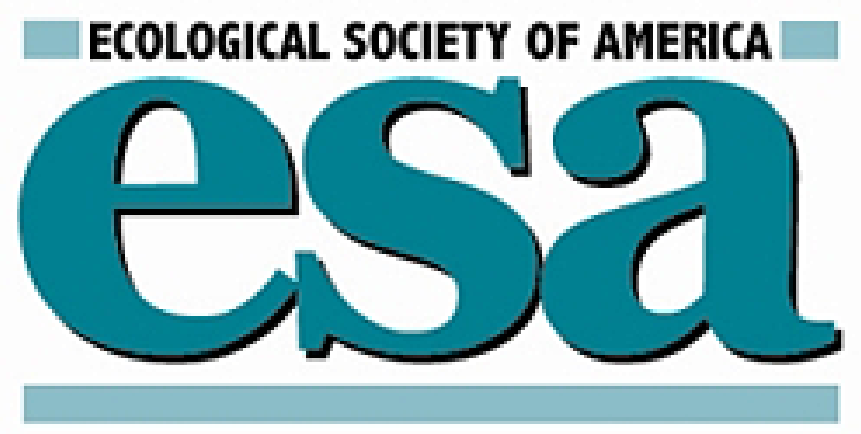

Characterizing the Spatial Structure of Songbird Cultures

Author(s): Paola Laiolo

Source: Ecological Applications, Vol. 18, No. 7 (Oct., 2008), pp. 1774-1780

Published by: Ecological Society of America

Stable URL: http://www.jstor.org/stable/40062250

Accessed: 06/05/2013 15:05

Your use of the JSTOR archive indicates your acceptance of the Terms \& Conditions of Use, available at http://www.jstor.org/page/info/about/policies/terms.jsp

JSTOR is a not-for-profit service that helps scholars, researchers, and students discover, use, and build upon a wide range of content in a trusted digital archive. We use information technology and tools to increase productivity and facilitate new forms of scholarship. For more information about JSTOR, please contact support@jstor.org. 


\title{
CHARACTERIZING THE SPATIAL STRUCTURE OF SONGBIRD CULTURES
}

\author{
Paola Laiolo ${ }^{1}$ \\ Estación Biológica de Doñana (CSIC), Avenida M. Luisa s/n, 41013 Sevilla and Instituto Cantábrico de Biodiversidad
}

(CSIC-UO-PA), Universidad de Oviedo, 33006 Oviedo, Spain

\begin{abstract}
Recent advances have shown that human-driven habitat transformations can affect the cultural attributes of animal populations in addition to their genetic integrity and dynamics. Here I propose using the song of oscine birds for identifying the cultural spatial structure of bird populations and highlighting critical thresholds associated with habitat fragmentation. I studied song variation over a wide geographical scale in a small and endangered passerine, the Dupont's Lark Chersophilus duponti, focusing on (1) cultural population structure, to determine a statistical representation of spatial variation in song and identify cultural units, and (2) the minimum patch size needed for an individual to develop a stable repertoire. I found that overall song diversity depends on variation among populations (beta-cultural diversity). Abrupt thresholds occurred in the relationships between individual song dissimilarity and geographic distance, and between individual song diversity and patch area. Spatial autocorrelation analysis showed that populations located as little as $5 \mathrm{~km}$ apart may have independently evolved their song traditions. Song diversity stabilized in patches as small as 100 ha supporting as few as $8-20$ males. Song repertoires of smaller patches were significantly poorer. Almost one-quarter of the study populations inhabited patches $<100$ ha, and their cultural traditions appear to have eroded. The analysis of spatial patterns in birdsong may be a useful tool for detecting subpopulations prone to extinction.
\end{abstract}

Key words: Chersophilus duponti; Dupont's Lark; habitat fragmentation; small populations; song diversity.

\section{INTRODUCTION}

One of the greatest goals of conservation biology is the conservation of small populations, which are often characteristic of threatened and endangered species (Caughley 1994). Inbreeding depression, demographic stochasticity, genetic drift, and Allee effects can combine to drive small populations into an extinction vortex (Gilpin and Soulé 1986, Lande and Shannon 1996). Habitat fragmentation and loss are among the most important anthropogenic threats to the viability of endangered species (Soule 1986). Although the concepts have occasionally been misused (Lindenmayer and Fischer 2007), many studies have demonstrated how the loss and isolation of habitat patches individuals from the landscape affects the dynamics, persistence, distribution, and genetic structure of populations (Andrén 1994, Wiegand et al. 2005).

Behaviors and learned traditions can be disrupted or lost when population size decreases in fragmented populations (Laiolo and Jovani 2007, Lonsdorf 2007). For instance, in elephants the loss of older, experienced individuals that model behavior for younger animals can affect social life and decrease group breeding success (McComb et al. 2001, Fox 2003). In African wild dogs,

Manuscript received 31 August 2007; revised 19 February 2008; accepted 13 March 2008. Corresponding Editor: T. R. Simona.

1 E-mail: paola@ebd.csic.es smaller packs show compromised cooperative behaviors (hunting, defense from kleptoparasitism, pup feeding, and babysitting), which affects individual fitness and population persistence (Courchamp et al. 2000, 2002, Courchamp and Macdonald 2001).

Although molecular tools are widely used to address conservation issues such as habitat destruction and changes in population size (Smith and Wayne 1996), few studies have attempted to quantify behavioral processes to determine the effects of habitat transformation on populations (Reed 1999). This is in part due to the difficulty of quantifying behaviors, and to the lack of statistical tools for quantifying spatial patterns of behavior. The song of oscine birds, one of the best studied cultural traits in nonhumans, could become a promising model for identifying the sensitivity of cultural subpopulations to habitat alterations (Laiolo and Tella 2007a). Birdsong can be split into a series of characters that are easy to quantify, such as repertoire size, song rate, spectro-temporal parameters, and other factors (Gil and Gahr 2002). Birdsong also varies geographically; dialects reflecting geographic song differences arise due to learning, either because of natural or anthropogenic barriers (Kroodsma 2004, Laiolo and Tella 2005, Podos and Warren 2007). Eventually, the evolution of birdsong is closely related to sexual selection, implying that it honestly indicates individual quality in intra-sexual competition and mate attraction (Gil and Gahr 2002). Such costly sexual traits can become good indicators of population status and 
viability because they reflect the condition of individuals (Reusch et al. 2001, Ahtiainen et al. 2004, Laiolo et al. 2008). Consequently, identifying cultural groups and the minimum habitat requirements for developing complete behavioral repertoires may improve conservation plans.

I studied song variation (namely variability in song repertoire) in a small and endangered passerine, the Dupont's Lark Chersophilus duponti, throughout its range in Spain. In Europe, the species is restricted to the remaining fragments of secondary steppelands of the Iberian Peninsula; its populations are patchily distributed, small, declining, and threatened by increasing habitat transformation (Tella et al. 2005, Laiolo and Tella 2006a). The communication system of this species is sensitive to habitat fragmentation both at local and regional scales (Laiolo and Tella 2005, 2006b). Laiolo and Tella (2007a) and Laiolo et al. (2008) found a positive correlation between Dupont's Lark song diversity and population size, productivity, and viability, and they suggested that poor individual repertoires can foreshadow population extinction.

Given the complexity of possible influences on song variation, the method used to obtain information on song variability may be critically important. I illustrate here two procedures accounting for acoustic variation associated to spatial patterns of isolation and patch size. First, I analyze the spatial autocorrelation of interindividual song dissimilarity. This approach is widely used to describe the spatial patterns of variation in molecular and phenotypic data, and to establish operational units for conservation (Sokal et al. 1998, Diniz-Filho and Telles 2002). Second, I address the minimum patch size needed for an individual to maintain stable song diversity, and I identify critical thresholds associated with habitat fragmentation. Findings are then used to quantify minimum habitat requirements from a cultural perspective, and approaches to applying these results to conservation are discussed.

\section{Methods}

In the period 2004-2007, I visited 51 steppe patches distributed throughout the distribution of Dupont's Lark in Spain, and recorded the songs from 385 males (Appendix A). Occupied patches varied greatly in size (from 20 to $>5000 \mathrm{ha}$ ) and distance to the nearest occupied fragment (from 4 to $80 \mathrm{~km}$ ), and in the size of the lark population (from 1 to $>250$ males) (Garza et al. 2005, Tella et al. 2005, Martin-Vivaldi et al. 2006, Seoane et al. 2006). The patchiness in distribution of Dupont's Larks is largely due to human-driven habitat transformation, which is threatening the species' persistence in many small and isolated areas (Tella et al. 2005, Laiolo and Tella 2007b). Three small populations out of the 51 studied $(6 \%)$ went extinct during this research (Tella et al. 2005, Laiolo et al. 2008).

Male songs were recorded in individual territories using a Sony TC-D8 DAT recorder (Sony, Tokyo, Japan) and a Sennheiser ME67 microphone (frequency response 50-20000 Hz; Sennheiser, Wedemark, Germany), and singer locations were established using a Garmin eTrex Navigator Global Positioning System (Garmin, Olathe, Kansas, USA).

Dupont's Lark song includes a variable number of discrete song types (equivalent to phrases or sequences in the birdsong literature), from two to 12 per individual, which are repeated and shared by neighboring males during spring disputes (details can be found in Laiolo and Tella 2005, and spectrograms in Appendix B). I characterized individual repertoires and classified song types by visually inspecting $>7600$ spectrograms using Avisoft SASLab Pro 3.91 software (Fast Fourier Transform, sampling frequency $22050 \mathrm{~Hz}$, FFT length 512 , time resolution $8.9 \mathrm{~ms}$, frequency resolution $43 \mathrm{~Hz}$; Window Function, Bartlett; Avisoft, Berlin, Germany). The classification criterion of Laiolo and Tella (2005) was followed: two song types had to match at least three-quarters of their component notes to be considered as the same, and the matching portions had to be similar in timing, frequency, and note shape (notes were classified as trills, tonal, with increasing, decreasing, or constant pitch). Song types were categorized blind, to assure consistency of classification. Once songs were classified, a binary matrix of individual $\times$ song type was built, scoring the presence (1) or absence ( 0 ) of a song type in the pool of each individual.

For each song type, the mean frequency within populations (i.e., among individuals of the same population) and its relative frequency among populations were calculated. These measures were then used to partition overall song diversity of Dupont's Lark into its within- and among-population components (Laiolo and Tella 2006a). I estimated song diversity within populations ( $\alpha$-diversity) using the Shannon index $\left(H^{\prime}=-\Sigma p_{i}\right.$ $\log \left(p_{i}\right)$, where $p_{i}$ is the frequency of song type $\left.i\right)$, and the overall diversity of the Iberian Peninsula ( $\gamma$-diversity). Song heterogeneity among populations ( $\beta$-diversity) resulted by subtracting mean $\alpha$-diversity from $\gamma$-diversity.

I derived a measure of the size of each steppe from the literature (Tella et al. 2005, Martin-Vivaldi et al. 2006, Seoane et al. 2006) and aerial orthophotographs (SIGPAC 2004).

A classical spatial autocorrelation approach was used to analyze song variation over distance. Using the individual $\times$ song type matrix, the squared Euclidean distances among each pair of individuals were estimated to generate a song dissimilarity matrix that included 73920 pairwise dissimilarities $(d)$. Pairwise geographic distances between recorded individuals were calculated and then grouped in bins $(h)$ with a lag distance of $1 \mathrm{~km}$. Individual pairs with separation $>100 \mathrm{~km}$ were grouped into only one bin (from 100 to $610 \mathrm{~km}$ ) because of low sample sizes for kilometer-wide bins at those lags (in keeping with Fleishman and Mac Nally 2006). The number of pairwise comparisons $\left(N_{h}\right)$ ranged from 28 to 


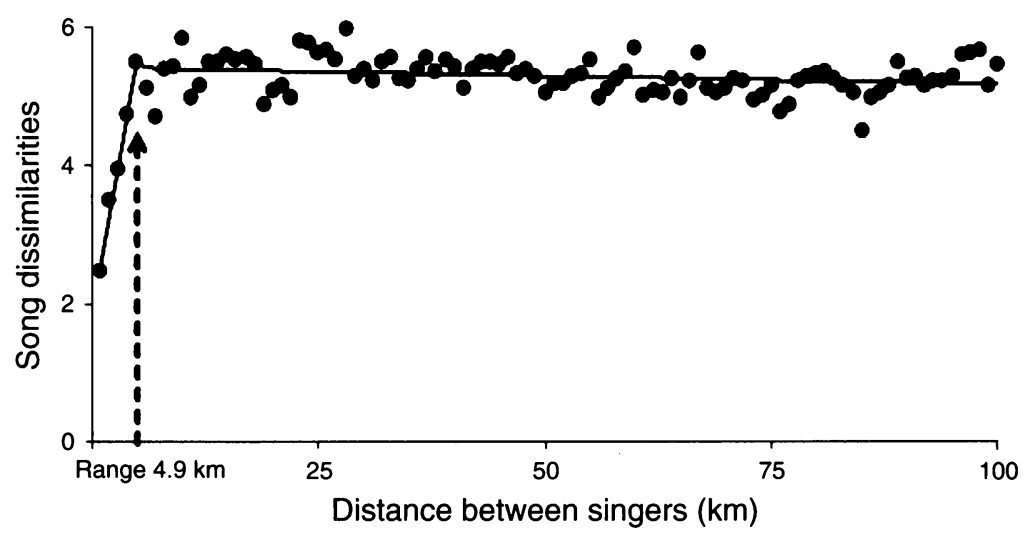

FIG. 1. Geographic structure of song variation: relationship between pairwise song dissimilarities and straight-line distances between singers. The best-fit piecewise model line and the critical threshold distance (dashed vertical line) are also shown; distance lags between 100 and $605 \mathrm{~km}$ were grouped in the last lag in the figure. Song dissimilarity refers to the number of song types that are not shared in the repertoires of individuals. Up to the distance of $4.9 \mathrm{~km}$, song dissimilarities increase with distance; for larger distances the number of song types that are not shared stabilizes to a maximum value. This value is approximately the number of song types in the repertoire of the individuals, i.e., birds do not share any song type for distances $>5 \mathrm{~km}$.

955 per bin below $100 \mathrm{~km}$, and summed 54121 in the last bin $(100-610 \mathrm{~km})$.

For each lag, the mean song dissimilarity was computed as

$$
\gamma_{h}=\frac{1}{2 N_{h}} \sum_{1=\alpha}^{N_{h}} d_{h} .
$$

In order to analyze song variation $(\gamma(h))$ over distance $(h)$, the data were fitted to the most commonly used functions in geostatistical analysis (Cressie 1993). I considered both models describing clinal trends, with dissimilarities increasing smoothly with geographic distance (linear and power-law models), and nonlinear threshold models in which spatial variation was discontinuous (exponential and piecewise models). In the exponential model, spatial autocorrelation only occurs within a certain lag distance (the range); it then reaches an asymptote (the sill) beyond which song pools are statistically uncorrelated and can be considered as independent (example in Fig. 2). The simplest piecewise regression model joins two straight lines at a breakpoint (the critical distance threshold; Toms and Lesperance 2003; example in Fig. 1). The magnitude and shape of the relationship between mean individual song diversity per patch (pool of song types of an individual) and patch size were also analyzed using the above statistical functions. Nonlinear least square regressions were used to fit all models and estimate the function parameters, and a criterion-based approach (Akaike Information Criterion, AIC; Akaike 1973) was followed to estimate the model of best fit.

\section{RESUlts}

A total of 253 different song types were recorded in the study area, and 169 of these were shared by at least two populations. Most of the diversity of Dupont's Lark song repertoire depended upon variation among popu- lations, which accounted for three-quarters of the diversity at the national scale ( $\beta$ song diversity $=$ $75.3 \%$ of song diversity in Spain). Common song types within a population were not more likely to be distributed among a large number of populations than uncommon song types, given that the frequency of a song type within a population was not related to its frequency among populations $\left(r^{2}=0.01, F_{1,251}=3.72\right.$, not significant).

When analyzing song variation through space, the best model fit for spatial song differentiation was a piecewise $\left(r^{2}=0.69, F_{4,96}=72.0, P<0.001, \mathrm{AIC}=13.9\right)$ and an exponential model $\left(r^{2}=0.66, F_{3,97}=93.2, P<\right.$ 0.001 , AIC $=14.5$ ); other models were separated by $>6$ AIC units from the fit of the best ones. As depicted in Fig. 1, song dissimilarities increased up to a distance of $4.9 \pm 0.4 \mathrm{~km}$ (mean $\pm \mathrm{SE}$ ) and leveled off for larger lags.

Individual song diversity stabilized when the occupied patches reached the size of 98 ha (exponential model: $r^{2}$ $=0.20, F_{3,48}=6.1, P=0.0044$, AIC $=54.25$ ); at this distance song repertoires reached the asymptotic value (sill) of 5.74 call types (Fig. 2). When patch size was virtually zero, intrinsic song diversity was 3.6 song types, the statistical minimum (nugget) achieved by an average male. Other models were separated by $>4.6$ AIC units from the fit of the exponential one. To further test for variation related to patch size and account for individual variation within patches, I performed randomization tests in one-way ANOVAs, reshuffling individual data on song repertoire between the two categories of patch size, below and above the threshold size of 98 ha. The program .EcoSim version 7.19 was used to repeat the process 10000 times and generate a mean and a variance for observed and expected values under random variation (Gotelli and Entsminger 2001). Individuals from patches smaller than the critical thresholds sang a significantly poorer repertoire than those expected by 


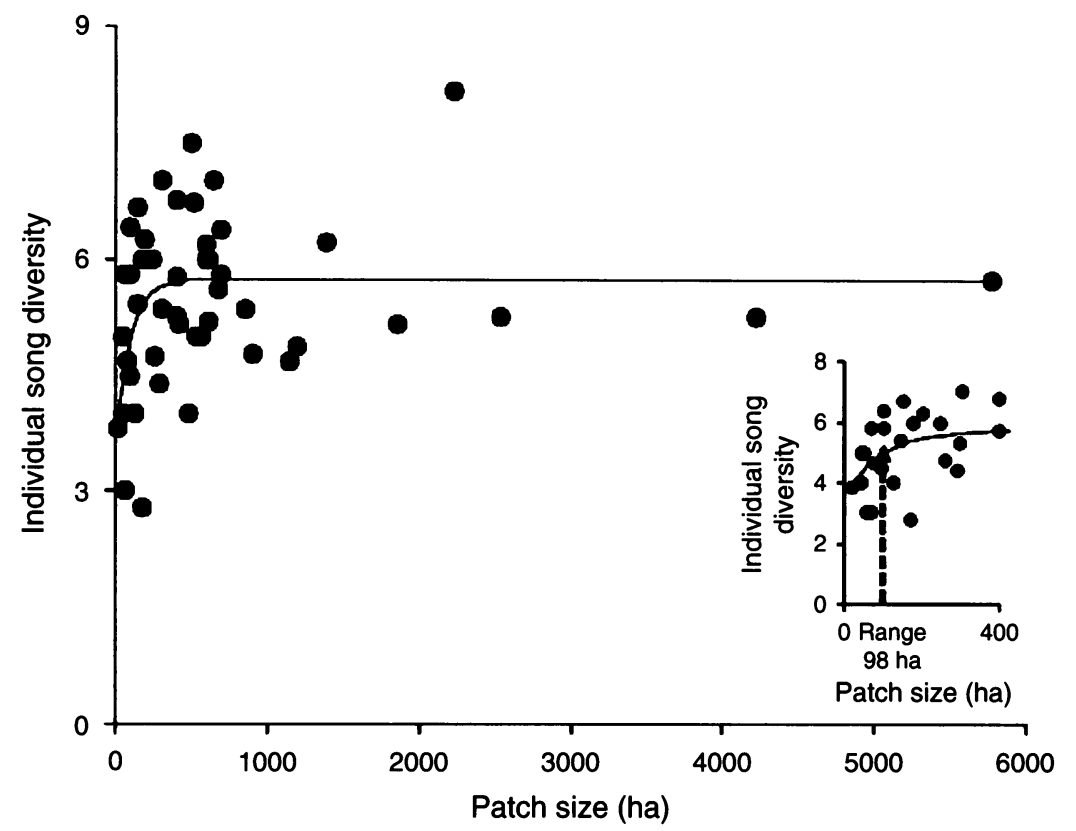

FIG. 2. Individual song diversity-area relationship. The inset graph represents variation at patch size $<400$ ha. The best-fit exponential model line and the critical threshold patch size for song diversity to stabilize (dashed vertical line) are also shown. Individual song diversity represents the number of different song types in the repertoire of the individual.

random sampling $(4.67 \pm 0.12$ song types vs. $5.38 \pm 0.16$ song types, mean $\left.\pm \mathrm{SE}, F_{1,384}=14.41, P<0.001\right)$. Almost one-quarter $(23.5 \%)$ of the study patches were below the minimum size threshold required for individual song repertoire to stabilize (Fig. 3).

\section{Discussion}

This study applied a geostatistical approach to a large sample of individuals and populations to identify the cultural population structure of birdsong in an endangered passerine. Understanding patterns of spatial variation in birdsong allowed determination of the extent to which individual cultural diversity depends upon the availability of suitable habitat.

The communication systems of Dupont's Lark have been found in previous studies to respond to anthropogenic habitat fragmentation both at the local scale (in the acoustic mechanisms of territorial defense; Laiolo and Tella 2005) and at the regional scale (in territorial call variation patterns; Laiolo and Tella 2006b), and to be sensitive to both population reduction and isolation among patches (Laiolo and Tella 2007a). The positive relationship between individual song diversity and the annual rate of population change (a measure of population viability; Laiolo et al. 2008) in Dupont's Lark point to the importance of identifying minimum thresholds of song diversity associated with minimum viable populations.

Critical and abrupt thresholds occur when modeling both song dissimilarity-distance and individual song diversity-area relationships. Song spatial autocorrelation is limited to lags shorter than $5 \mathrm{~km}$, within which similarity between song pools decreases linearly with distances. Exceeding this lag, dissimilarities stabilize. Fig. 1 suggests that each habitat fragment evolves its song pool in a process that appears independent of the distance between patches. Individuals 10 or $100 \mathrm{~km}$ apart roughly show the same song dissimilarities. The independent development of songs among patches is also confirmed by the analysis of song type frequencies within and among fragments. If song traditions flow quickly among fragments with their bearers, song types that occur at high frequency within populations are expected to be found in several populations (Lynch and Baker 1994). In Dupont's Lark, where song is learned

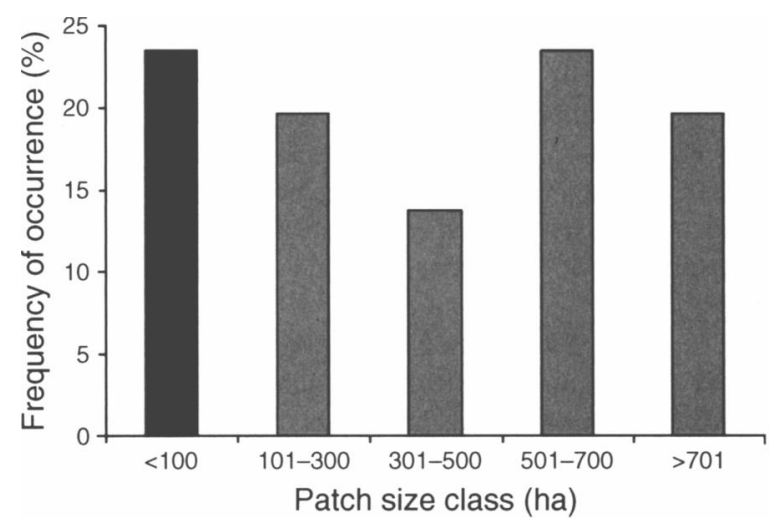

FIG. 3. Frequency distribution of steppe patch sizes in the study area. Almost one-quarter of the subpopulation (black bar) occurs in patches smaller than the threshold value for individual song diversity to stabilize. 
after dispersal, within- and among-population song frequencies are unrelated. The tendency of copying song types and hence contributing to song type/cultural transmission is strong only in nearby birds only. Laiolo and Tella (2005) found out that neighbor males shared a larger percentage of song types than birds that were not neighbors, and the phenomenon was more prevalent when patches of suitable habitat were isolated. Song transmission among individuals also depends on the dispersal of adult males, which are the reservoir of song traditions. If short distances are moved, song units are likely to have limited dissemination (Laiolo and Tella 2007a). The maximum dispersal distance that has been documented for the Dupont's Lark adult male is slightly below the measured threshold of $3 \mathrm{~km}$ in the Iberian system (Martin-Vivaldi et al. 2006; V. Garza and F. Suarez, unpublished data) and $2.2 \mathrm{~km}$ in the more fragmented Ebro Valley (Laiolo et al. 2007). Another consequence of this song differentiation pattern is that most of the song diversity within the Iberian Peninsula depends upon variation among populations ( $\beta$ song diversity represents three-quarters of the national song pool).

Patterns of spatial autocorrelation suggest that any two populations separated by distances $>5 \mathrm{~km}$ may have independently evolved cultural traits. When considering each song unit as a separate cultural trait, it is likely that most song variation among populations is neutral (Slater 1986, Whitehead et al. 2004). The occurrence of, say, song type A rather than song type $B$ in a population should not give males an adaptive gain, unless song units confer an advantage in reproduction, as in the case of specific syllables used for malemale competition (Saino et al. 2003) or mate attraction ("sexy" syllables; Vallet et al. 1997). It has been shown that new song types can rapidly develop and disappear in some passerine species, suggesting that some variation among populations has no adaptive significance (Kopuchian et al. 2004). On the other hand, Ruegg et al. (2006) found that dialects in Swainson's Thrush (Catharus ustulatus) might have been shaped by selection favoring spectral characteristics that promote sound transmission in different habitat types. In the latter example, variation is adaptive and reflects ecological differences among populations. In Dupont's Lark, there is no evidence that specific song types benefit individual Dupont's Lark males or that they favor sound transmission in different habitats (Laiolo and Tella 2007b).

The cultural diversity-area relationship found in this study highlights a critical patch size below which individual song diversity starts eroding. This threshold appears to occur at patch densities of 8-20 males per 100 ha. Although population extinctions have occurred with greater frequency in small $(<100 \mathrm{ha})$ than in large areas of steppe during the last decades (Tella et al. 2005), average song repertoires of less than five song types may indicate population units that require active manage- ment. Moreover, individual song diversity is linked to population,viability because of its positive association with population size (determining the social milieu in which repertoires develop) and with population productivity (through a possible link with reproductive success). No relationship with individual survival has been demonstrated (Laiolo et al. 2008). The goal of a conservation plan centered on cultural traits should therefore be the maintenance of a viable cultural patrimony through the conservation of adequate patches of steppe habitats ( $>100$ ha), necessary to preserve song variation and population viability. In the specific case of songs, maintaining patch connectivity may be less relevant than stopping habitat loss to preserve song diversity, given the short lags at which song repertoire shifts.

This study suggests that Dupont's Lark needs enough suitable habitat to develop an original and rich cultural repertoire. While the threshold patch size found in this study may not be relevant for widespread bird species with continuous ranges, it may be critical for species that persist in small isolated populations. In the case of Dupont's Lark, almost $25 \%$ of the populations occupied smaller steppe patches that showed signs of eroding cultural diversity.

Although fluctuations in the song pools of populations might be expected due to the fast evolving nature of culture (Cavalli-Sforza and Feldman 1981), it is unlikely that these changes could affect the thresholds found in this study at the individual level, as song repertoire is constrained to a maximum and minimum number of types per individual in Dupont's Lark. The highest individual threshold was not exceeded even when the population cultural background was far richer than 12 song types, the maximum for an individual (population repertoire sizes can reach up to 50 song types; Laiolo and Tella 2007a). On the other hand, individuals from small populations increase their song pool through heterospecific song imitations when no conspecifics are available as tutors (Laiolo et al. 2008), thus preventing excessive drops in the number of song types. This study addressed the entire European population of Dupont's Lark, which represents $25-50 \%$ of the estimated global population of the species (BirdLife International 2005). Therefore, in spite of differences in the history of habitat alteration in different areas (Laiolo and Tella 2005) and potential oscillations due to fast cultural evolution, the habitat size thresholds found here could be consistent across the species' range.

In conclusion, I propose that spatial analyses of bioacoustic data (landscape bioacoustics), still a rather novel approach in conservation biology (Laiolo and Tella 2006b), may be a useful tool for detecting segments of bird populations with low viability. An individualbased approach based on spatial analyses of song traits can be carried out even when population sizes are naturally extremely low (2-10 individuals), and studies based on population genetics may not be feasible. 
Although studies of the linkages between song diversity and individual fitness permeate much of behavioral ecology, I believe understanding relationships between song diversity and population viability is worthy of future exploration and may provide useful insights to questions in applied avian ecology and conservation.

\section{ACKNOWLEDGMents}

I am grateful to J. L. Tella, M. Vögeli, D. Serrano, V. Garza, F. Suárez, J. Manrique, and P. Arratibel for kindly providing information on Dupont's Lark localizations in Spain, and to M. Vögeli, M. Mendez, D. Serrano, J. L. Tella, X. Picó, and P. Jordano for discussing with me the results of this research. I also thank three anonymous referees, A. McCallum, and T. Simons for critically and constructively commenting on an early version of the manuscript. During this study, I was supported by a Marie Curie Intra-European Fellowship within the 6th European Framework Program (2004-2005), an Excellence Project of the Junta the Andalucia to J. L. Tella (2006), and a Ramon y Cajal contract of the Spanish Ministry of Education and Science (2007).

\section{Literature Cited}

$\rightarrow$ Ahtiainen, J. J., R. V. Alatalo, J. Mappes, and L. Vertainen. 2004. Decreased sexual signalling reveals reduced viability in small populations of the drumming wolf spider Hygrolycosa rubrofasciata. Proceedings of the Royal Society of London B 271:1839-1845

Akaike, H. 1973. Information theory as an extension of the maximum likelihood principle. Pages 267-281 in B. N. Petrov and F. Csaksi, editors. Second International Symposium on Information Theory. Akademiai Kiado, Budapest, Hungary.

Andrén, H. 1994. Effects of habitat fragmentation on birds anc mammals in landscapes with different proportions of suitable habitat: a review. Oikos 71:355-366.

BirdLife International. 2005. Chersophilus duponti. In IUCN 2007. Red list of threatened species. International Union for Conservation of Nature and Natural Resources, Cambridge, UK. 〈http://www.iucnredlist.org

Caughley, G. 1994. Directions in conservation biology. Journal of Animal Ecology 63:215-244.

Cavalli-Sforza, L. L., and M. W. Feldman. 1981. Cultural transmission and evolution: a quantitative approach. Princeton University Press, Princeton, New Jersey, USA.

Courchamp, F., T. H. Clutton-Brock, and B. T. Grenfell. 2000. Multi-group dynamics and Allee effects in the African wild dog Lycaon pictus. Animal Conservation 3:277-285.

Courchamp, F., and D. W. Macdonald. 2001. Crucial importance of pack size in the African wild dog Lycaon pictus. Animal Conservation 4:169-174.

Courchamp, F., G. S. A. Rasmussen, and D. W. Macdonald. 2002. Small pack size imposes a trade-off between hunting and pop-guarding in the painted hunting dog Lycaon pictus. Behavioral Ecology 13:20-27.

Cressie, N. A. C. 1993. Statistics for spatial data. Wiley, New York, New York, USA.

Diniz-Filho, J. A. F., and M. P. Telles. 2002. Spatial autocorrelation analysis and the identification of operational units for conservation in continuous populations. Conservation Biology 16:924-935.

$\rightarrow$ Fleishman, E., and R. Mac Nally. 2006. Patterns of spatial autocorrelation of assemblages of birds, floristics, physiognomy, and primary productivity in the central Great Basin, USA. Diversity and Distributions 12:236-243.

Fox, D. 2003. More than meets the eye: behaviour and conservation. Conservation in Practice 4:20-29.

Garza, V., F. Suárez, J. Herranz, J. Traba, E. G. De La Morena, M. B. Morales, R. González, and M. Castañeda. 2005. Space use and habitat selection of the endangered
Dupont's Lark in Spain: the breeding and postbreeding periods. Ardeola 52:133-146.

Gil, D., and M. Gahr. 2002. The honesty of bird song: multiple constraints for multiple traits. Trends in Ecology and Evolution 17:133-141.

Gilpin, M. E., and M. E. Soulé. 1986. Minimum viable populations: processes of species extinctions. Pages 19-23 in M. E. Soule, editor. Conservation biology: the science of scarcity and diversity. Sinauer Associates, Sunderland, Massachusetts, USA.

Gotelli, N. J., and G. L. Entsminger. 2001. EcoSim: null models software for ecology. Version 7.0. Acquired Intelligence and Kesey-Bear, Burlington, Vermont, USA.

Kopuchian, C., D. A. Lijtmaer, P. L. Tubaro, and P. Handford. 2004. Temporal stability and change in a microgeographical pattern of song variation in the rufous-collared sparrow. Animal Behaviour 68:551-559.

Kroodsma, D. 2004. The diversity and plasticity of birdsong. Pages 108-131 in P. Marler and H. Slabbekoorn, editors. Nature's music: the science of birdsong. Elsevier Academic Press, San Diego, California, USA.

Laiolo, P., and R. Jovani. 2007. The emergence of animal culture conservation. Trends in Ecology and Evolution 22:5.

$\rightarrow$ Laiolo, P., and J. L. Tella. 2005. Habitat fragmentation affects culture transmission: patterns of song matching in Dupont's lark. Journal of Applied Ecology 42:1183-1193.

Laiolo, P., and J. L. Tella. 2006a. Fate of unproductive and unattractive habitats: recent changes in Iberian steppes and their effects on endangered avifauna. Environmental Conservation 33:223-232.

Laiolo, P., and J. L. Tella. 2006b. Landscape bioacoustics allows detection of the effects of habitat patchiness on population structure. Ecology 87:1203-1214.

Laiolo, P., and J. L. Tella. 2007a. The erosion of animal cultures in fragmented landscapes. Frontiers in Ecology and the Environment 5:68-72.

Laiolo, P., and J. L. Tella. 2007b. Vocal diversity patterns. Reply. Frontiers in Ecology and the Environment 5:406-407.

Laiolo, P., M. Vögeli, D. Serrano, and J. L. Tella. 2007. Testing acoustic versus physical marking: two complementary methods for individual-based monitoring of elusive species. Journal of Avian Biology 38:672-681.

Laiolo, P., M. Vögeli, D. Serrano, and J. L. Tella. 2008. Song diversity predicts the viability of fragmented bird populations. PLoS ONE 3(3):e1822.

Lande, R., and S. Shannon. 1996. The role of genetic variation in adaptation and population persistence in a changing environment. Evolution 50:434-437.

Lindenmayer, D. B., and J. Fischer. 2007. Tackling the habitat fragmentation panchreston. Trends in Ecology and Evolution 22:127-132.

Lonsdorf, V. 2007. The role of behavioral research in the conservation of chimpanzees and gorillas. Journal of Applied Animal Welfare Science 10:71-78.

Lynch, A., and A. J. Baker. 1994. A population memetics approach to cultural evolution in chaffinch song: differentiation among populations. Evolution 48:351-359.

Martín-Vivaldi, M., et al. 2006. Determinación del área de distribución de la alondra de Dupont (Chersophilus duponti) en la población de El Padul (Granada). Acta Granatense, Universidad de Granada, Spain.

McComb, K., C. Moss, S. Durant, L. Baker, and S. Sayialel. 2001. Matriarchs act as repositories of social knowledge in African elephants. Science 292:491-494.

Podos, J., and P. S. Warren. 2007. The evolution of geographic variation in birdsong. Advances in the Study of Behavior 37: 404-458.

Reed, J. M. 1999. The role of behavior in recent avian extinctions and endangerments. Conservation Biology 13: 232-241. 
Reusch, T. B. H., M. A. Haberli, P. B. Aeschlimann, and M. Milinski. 2001. Female sticklebacks count alleles in a strategy of sexual selection explaining MHC polymorphism. Nature 414:300-302.

Ruegg, K., H. Slabbekoorn, S. Clegg, and T. B. Smith. 2006. Divergence in mating signals correlates with ecological variation in the migratory songbird, Swainson's thrush (Catharus ustulatus). Molecular Ecology 15:3147-3156.

$\rightarrow$ Saino, N., M. Romano, R. Sacchi, P. Ninni, P. Galeotti, and A. P. Møller. 2003. Do male barn swallows (Hirundo rustica) experience a trade-off between the expression of multiple sexual signals? Behavioral Ecology and Sociobiology 54:465471.

Seoane, J., J. Justribó, F. Garcia, J. Retamar, C. Raban, and J. C. Atienza. 2006. Habitat-suitability modelling to assess the effects of land-use changes on Dupont's lark Chersophilus duponti: a case study in the Layna Important Bird Area. Biological Conservation 128:241-252.

SIG-PAC. 2004. Sistema de identificación geográfica de parcelas agrícolas. Ministerio de Agricoltura, Pesca y Alimentación, Madrid, Spain. /http://sigpac.mapa.es/fega/ visor/>

Slater, P. J. B. 1986. Cultural transmission of bird song. Trends in Ecology and Evolution 1:94-97.
Smith, T. B., and R. K. Wayne. 1996. Molecular genetic approaches in conservation. Oxford University Press, New York, New York, USA.

Sokal, R. R., N. L. Oden, and B. A. Thomson. 1998. Local spatial autocorrelation in biological variables. Biological Journal of the Linnean Society 65:41-62.

Soule, M. E. 1986. What is conservation biology? BioScience 35:727-734.

Tella, J. L., M. Vögeli, D. Serrano, and M. Carrete. 2005. Current status of the endangered Dupont's lark in Spain: overestimation, decline, and extinction of local populations. Oryx 39:90-94.

Toms, J. D., and M. L. Lesperance. 2003. Piecewise regression: a tool for identifying ecological thresholds. Ecology 84: 2034-2041.

Vallet, E. M., M. L. Kreutzer, I. Beme, and L. Kiosseva. 1997. 'Sexy' syllables in male canary songs: honest signals of motor constraints on male vocal production? Advances in Ethology 32:132.

Whitehead, H., L. Rendell, R. W. Osborne, and B. Wursig. 2004. Culture and conservation of non-humans with reference to whales and dolphins: review and new directions. Biological Conservation 120:427-437.

Wiegand, T., E. Revilla, and K. A. Moloney. 2005. Effects of habitat loss and fragmentation on population dynamics. Conservation Biology 19:108-121.

\section{APPENDIX A}

A map representing the study populations and the distribution of Dupont's Lark in Spain (Ecological Archives A018-061-A1).

\section{APPENDIX B}

A spectrogram of the song of two Dupont's Lark males, one from a 2000-ha fragment and another from a 100-ha fragment (Ecological Archives A018-061-A2). 